\title{
LA ACCIÓN SOCIAL EN LA UNIVERSIDAD PÚBLICA: ACTUANDO EN LA ERA NEOLIBERAL
}

\section{SOCIAL ACTION IN PUBLIC UNIVERSITY: ACTING IN NEOLIBERAL ERA}

Qué tal si deliramos por un ratito qué tal si clavamos los ojos más allá de la infamia para adivinar otro mundo posible.

Eduardo Galeano

\author{
Flavio Mora Moraga* \\ Ana Lucía Gutiérrez Espeleta**
}

\section{RESUMEN}

En la academia latinoamericana se debate sobre el papel de las universidades públicas frente a la realidad social y el papel del neoliberalismo en las mismas, siendo algunas de estas, administradas y orientadas con principios del libre mercado. En este contexto, es fundamental definir la acción social y su papel dentro de las universidades públicas como parte del compromiso social con las comunidades y con la sociedad. Para ello se revisa las principales transformaciones que han sufrido las universidades públicas en el contexto de libre mercado. Este artículo, intenta aportar a este debate a partir de una experiencia de acción social en una comunidad de Limón, Costa Rica.

PALABRAS CLAVE: UNIVERSIDADES * ENSEÑANZA PÚBLICA * NEOLIBERALISMO * ACCIÓN SOCIAL * COMUNIDAD

\section{ABSTRACT}

In the Latin America academy debate about the role of public universities against the social reality and the role of neoliberalism, with some of them, managed and directed with free market principles. In this context, it is essential to define social action and its role in public universities as part of social commitment to the community and society. For this, we

\footnotetext{
* Escuela de Ciencias Políticas de la Universidad de Costa Rica (UCR). jf2m013@gmail.com

** Investigadora pensionada de la Universidad de Costa Rica (UCR). ana.gutierrez@ucr.ac.cr
} 
review the main changes that have been public universities in the context of free market. This article attempts to contribute to this debate from one experience of social action in a community of Limon, Costa Rica. KEYWORDS: UNIVERSITIES * PUBLIC EDUCATION * NEOLIBERALISM * SOCIAL ACTION *
COMMUNITY

\section{INTRODUCCIÓN}

El debate actual en la academia latinoamericana contempla el papel que cumplen las universidades públicas ${ }^{1}$ frente a la realidad social, es por esto que la generación de conocimiento y la pregunta cómo y desde dónde se genera este conocimiento toma importancia en esta discusión.

El papel de la Universidad como ente investigador y como actor de la misma realidad no son parte de una nueva discusión, sino que lleva varias décadas, pero es necesario examinar qué y por qué se está haciendo investigación; asimismo, el papel que implican estas investigaciones en la realidad social y en las distintas comunidades en las que la Universidad está presente.

De la misma manera, surge la pregunta de si una universidad pública debe comprometerse con la transformación social. Desde la percepción de los autores, la respuesta es que por sus orígenes, así debe ser. Una institución de este tipo fue pensada para garantizar el derecho a la educación de todas las personas, con el objetivo de brindar un aprendizaje de calidad $y$ que se encuentre siempre en constante discusión, de manera que permita el fortalecimiento de la crítica social para "la consecución de una justicia social, de equidad, del desarrollo integral, de la libertad plena y de la total independencia de nuestro pueblo" (Universidad de Costa Rica-ucR, 1974: 1).

1 Cuando se hace referencia a universidad pública, se piensa en la universidad pública latinoamericana y concretamente, la Universidad de Costa Rica, en la cual se cincunscribe el proyecto de acción social que motiva esta reflexión.
La transformación social es, o al menos debería ser, uno de los objetivos de la universidad pública, pero la pérdida de prioridad de la universidad pública en las políticas del Estado, fue resultado de la pérdida general de prioridades de las políticas sociales para la década de los 90 , en pleno apogeo del modelo de desarrollo neoliberal y de los planes de integración regional, justificando la explotación comercial de la educación y por lo tanto, de las universidades (De Sousa Santos, 2004).

La acción social ${ }^{2}$ es una perspectiva de trabajo con la gente al servicio de esta transformación, pero en la realidad actual de la educación pública, no necesariamente es una prioridad, por ello, es importante repensar el papel de la acción social dentro del modelo presente de universidad pública.

Esta reflexión surge a partir del proyecto de acción social "Apoyo al fortalecimiento de la organización comunal amuda para la defensa de los recursos naturales", llevado a cabo en el Instituto de Investigaciones Sociales de la Universidad de Costa Rica (UCR), durante julio de 2011 a junio de 2013, en el cual se trabajó con la Asociación de Mujeres para el Desarrollo de África (амиDA), en la localidad de África de Guácimo en la provincia de Limón. El mismo

2 Cuando se habla de acción social, se refiere al proceso de construcción de conocimiento a partir de una gran diversidad de saberes. Contraria a la idea de la acción social como un asistencialismo donde el único conocimiento válido es el académico. El término investigación acción describe una forma de investigación que puede ligar el quehacer universitario con programas de acción social (construcción conjunta de conocimiento) de manera que responda a la realidad social en búsqueda de soluciones y cuya finalidad es la organización para realizar acciones que transformen dicha realidad. 
se planteó como objetivo principal el apoyar el fortalecimiento de AMUDA como organización comunal que defiende y pretende vivir en armonía con el ambiente.

Primeramente, se mostrará las principales transformaciones que han sufrido las universidades públicas en el marco del libre mercado; segundo, se analizará la importancia de la acción social dentro de la educación superior pública como parte de su compromiso social en el contexto citado anteriormente $y$ finalmente, se compartirá la experiencia en AмuDA.

\section{¿COMPROMISO SOCIAL? LA UNIVERSIDAD PÚBLICA EN EL CONTEXTO DEL LIBRE MERCADO}

\section{La educación popular es la dimensión educativa de la acción política Oscar Jara}

En el auge del modelo de desarrollo neoliberal, que toma fuerza en los 70 y su mayor apogeo en la década de los 90 , donde las instituciones públicas sufrieron una desregulación ${ }^{3}$, que provocó que su objetivo primordial fuera las de ser socias de la empresa privada (De Sousa Santos, 2006), motivando un emprendedurismo social (Lammerink, 1999) y la transformación de ciudadanos en inversionistas, esto sumado al auge de las transnacionales, gracias a la apertura comercial.

Lo anterior generó en la educación superior una "crisis institucional: la idea de que, por un lado, la universidad se creó en la autonomía $y$ ahora se busca, cada vez más, que sea conducida y administrada como una empresa, con criterios de eficacia que son típicos del mundo empresarial" (De Sousa Santos, 2006: 59). Esto enmarcado dentro de un mercado de servicios, donde los servicios más rentables son los que son considerados como necesarios y a los que se les destinará mayores recursos.

Se debe entender que la constitución de las universidades en América Latina estuvo muy relacionada con la búsqueda de un mejor

3 Sobre todo de espacios que ya se habían "ganado" como la acción social o extensión. Además de espacios que no son "útiles", que no son "eficientes", que no generan ganancias económicas. status de la "clase" media y alta; aún así, se configuraban también como espacios a los que las clases populares podrían acceder. En la década de los 50 se regían por la búsqueda del producto cuantificable (Chávez y Daza, 2003), lo que implicaba una lejanía entre las comunidades "investigadas" y aquellos que las investigaban con sus títulos que reflejaban un status especial.

Para la década de los 60 y 70, los movimientos populares toman auge dentro de las universidades públicas donde impulsa la "intervención para comprender y cambiar la realidad social" (Chávez y Daza, 2003: 119); cumpliendo un objetivo de una institución de educación superior pública a través de proyectos orientados a unir teoría y práctica.

En el caso de Costa Rica, la educación superior pública nace desde inicios de los 1800 s, se convierte en una educación del Valle Central y de una población que había logrado acumular cierta riqueza, que buscan cierta integración y la construcción de una identidad nacional dentro del proyecto liberal-democrático (González, 1999).

En estos países, la universidad pública $-y$ el sistema educativo en su conjunto- estuvo siempre ligada a la construcción del proyecto de nación, un proyecto nacional casi siempre elitista que la universidad debía formar. Se trataba de concebir proyectos nacionales de desarrollo o de modernización protagonizados por el Estado que buscaban crear o profundizar la coherencia y la cohesión del país como espacio económico, social y cultural (De Sousa Santos, 2004: 29).

En la década de los 70 en la Universidad de Costa Rica, los estudiantes deciden organizarse, ser parte de las discusiones sobre la Universidad, tanto en organización como en contenido y es así como, para el III Congreso Universitario de la ucr, en el año de 1972, se plantean siete ejes de discusión: fines de la educación superior, Universidad y Sociedad, crecimiento de la Universidad, principios orientadores, gobierno y administración, hacienda universitaria y vida estudiantil. Dentro de estos ejes, la discusión permite que 
se plantee la creación de una Vicerrectoría de Acción Social, que se plasma en 1974:

Cuyo mandato fue constituirse en la instancia responsable de todas las formas de vinculación de la Universidad con la sociedad, con el fin de poner la capacidad académica universitaria al servicio de la nación. La acción social se legitima, entonces, como actividad fundamental, como el mecanismo que promueve y facilita la vinculación Universidad-Sociedad (Pérez y Meoño, 2006: 31).

Es importante destacar que la ucr se diferencia del resto de universidades públicas, ya que estás "optan por la extensión, la ucr se compromete con la acción social (...) que implica una relación de doble vía (...) que no promueve una actitud benefactora, asistencialista, de caridad, sino un intercambio de saberes populares, empíricos y académicos" (Pérez y Meoño, 2006: 33); de esta forma, la ucr delimita principios básicos de lo que se define como acción social donde "el otro (a) también colabora y participa; que implica compromiso y praxis mediante acciones concretas" (ídem: 34). De esta manera es evidente el modelo de acción social que se define en el discurso.

En la década de 1980, los ajustes estructurales trajeron consigo la reducción de presupuestos (Chávez y Daza, 2003), lo que dio inicio al proceso de "mercantilización" de las universidades públicas, una forma incipiente de enfocar los presupuestos hacia las necesidades del mercado más que en función de la sociedad. Esta "crisis económica" se extendió a la siguiente década, lo que provocó un crecimiento mucho más lento en la investigación $y$ condiciones casi inexistentes para la acción social (gráfico 1), el diseño de planes de estudio dirigidos a solventar la demanda del mercado y de procesos de acreditación que "uniformaban" la enseñanza y las pedagogías que la educación "requería".

\section{GRÁFICO 1 \\ PRESUPUESTO UNIVERSITARIO FONDOS TOTALES \\ 1974-2013}

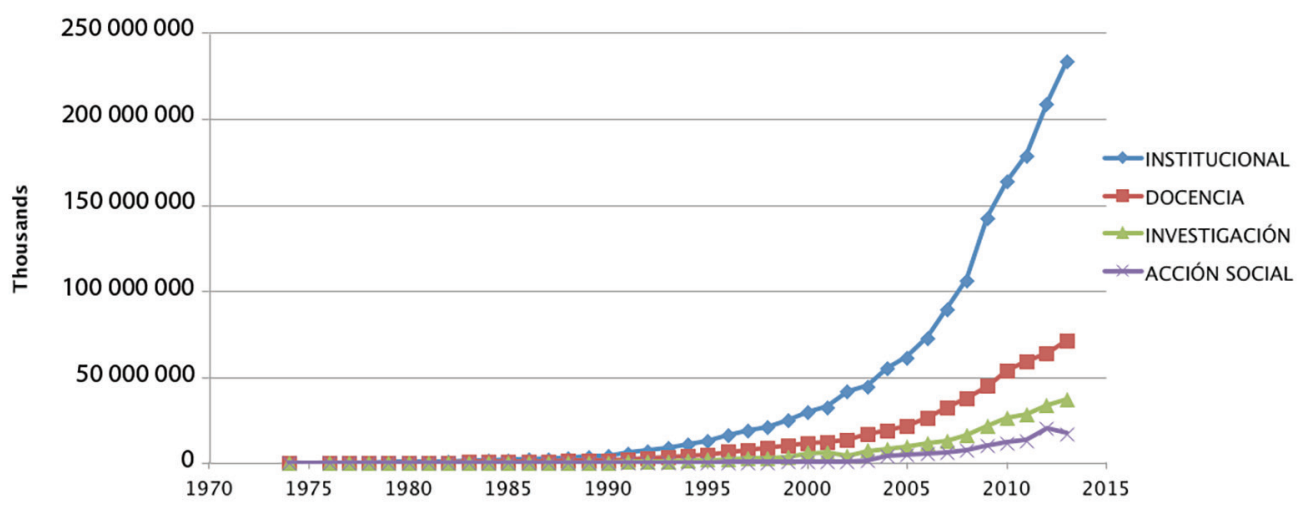

Fuente: Universidad de Costa Rica, Oficina de Planificación Universitaria (OPLAU), $2013^{4}$.

4 Se agradece la colaboración de la Oficina de Planificación Universitaria (OPLAU), por proporcionar la información necesaria para completar el gráfico 1. 
Como se observa en el gráfico 1 , el crecimiento del presupuesto institucional ha sido mucho mayor que el crecimiento en el financiamiento de las vicerrectorías de Docencia, Investigación y Acción Social; aun así, docencia ha mantenido un crecimiento paulatino, mientras que la Investigación y la Acción Social ha tenido un crecimiento mucho más lento y en ocasiones se ha estancado.
En el gráfico 2 se observa como la Vicerrectoría de Acción Social ha recibido en promedio un $3,72 \%$ del presupuesto institucional desde el año de su creación. Si bien es cierto, el presupuesto no es el único indicador para ver el apoyo institucional a una vicerrectoría, puede ser visto como un parámetro para ver como el financiamiento a esta vicerrectoría ha sido mucho menor que el de los otros pilares institucionales.

\section{GRÁFICO 2 \\ PROMEDIO PRESUPUESTO UNIVERSIDAD DE COSTA RICA \\ 1974-2013}

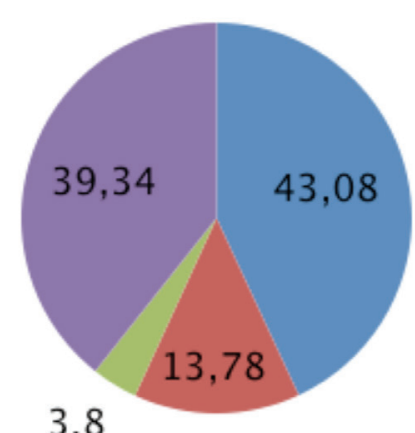

3,8
Docencia

- Investigación

- Acción Social

- Resto del presupuesto

Fuente: Universidad de Costa Rica, Oficina de Planificación Universitaria (OPLAU), 2013.

Dentro de este contexto, cabe preguntarse cuál es el modelo de Universidad que se quiere: el de un verdadero compromiso ${ }^{5}$ con la

$5 \quad$ El compromiso social tal y como lo plantea De Sousa Santos, implica una justicia de los conocimientos: "de los que están dentro y de los que están fuera de la universidad, no es que todo es válido para todo, pero hay que reconocer que hay conocimientos que circulan fuera de la universidad que son importantes y que la universidad está en una situación muy difícil en ellos: primero, fue entrenada para desconocer esos saberes; segundo, aunque hoy algunos gustarían de conocerlos, nuestro modelo de conocer algo, de reconocer algo, es certificar, $y$ no se puede certificar esos conocimientos; entonces tú no puedes ignorarlos, pero tampoco puedes certificarlos, $y$ eso ha creado tensiones muy interesantes en lo que llamo el área de las ecologías de saberes" (2011: 38). transformación de la sociedad hacia una más justa $y$ equitativa (entendiendo que a lo interno de la misma Universidad debe existir un debate continuo sobre cuál es la transformación social y cuál es el aporte que desde la Universidad puede llevarse a cabo), o una al servicio del mercado ${ }^{6}$ y cuyo impacto social es la venta

$6 \quad$ Este es un fenómeno que está sucediendo en todo el planeta: "En la periferia, donde la búsqueda de ingresos alternativos en el mercado o fuera de él es virtualmente imposible, la crisis logra proporciones catastróficas. Obviamente que los males venían de atrás, pero se agravaron mucho más en la última década con la crisis financiera del Estado y los programas de ajuste estructural. Un documento de la Unesco de 1997 acerca de la mayoría de las universidades en África, relataba un cuadro dramático de carencias de todo tipo: 
de servicios que busca - en el mejor de los casos-mejorar las condiciones de las personas sin transformar la "realidad".

Es entonces válido preguntarse, si las luchas por el acceso a la educación fueron concebidas para que esta educación fuera en detrimento de las mismas luchas sociales que buscan una sociedad más equitativa, lo que haría entonces a la misma educación concebida, más bien, hacia las necesidades de mano de obra mejor calificada dentro de una sociedad de maquilas, donde las personas son reemplazables por otras cada vez más "calificadas". Cuando más bien, si se parte de los distintos estatutos y congresos universitarios, las universidades están pensadas para lo contrario, están para contrarrestar la mediocridad de los medios de comunicación que fomentan la cultura chatarra, desinforman y manipulan dolosamente a la opinión ciudadana; implica también enfrentar la visión que pretende una educación universitaria exclusiva para la elite del dinero, excluyente de la inmensa mayoría de la población en edad de cursar estudios superiores y que considera que en los planes de estudio de las universidades solo deben incluirse temas y problemas que se consideran "técnicos", "útiles", "actualizados" y "políticamente correctos" (González, 2004).

... por un lado, el crecimiento de la mano de obra calificada ligada a la economía basada en el conocimiento y por

colapso de las infraestructuras, ausencia casi total de equipamiento, personal docente míseramente remunerado, $y$ por eso, desmotivado y propenso a la corrupción, poca o nula inversión en investigación. El Banco Mundial diagnosticó de modo semejante la situación y la declaró irremediable. Incapaz de incluir en sus cálculos la importancia de la universidad en la construcción de los proyectos de país y en la creación de pensamiento crítico $y$ de largo plazo, el Banco entendió que las universidades africanas no generaban suficiente "retorno". Consecuentemente, le impuso a los países africanos que dejaran de invertir en la universidad, concentrando sus pocos recursos en la educación primaria y secundaria, $y$ permitiendo que el mercado global de educación superior le resolviera el problema de la universidad" (De Sousa Santos, 2004: 17). otro lado, al crecimiento explosivo de un empleo con bajísimo nivel de calificación. La globalización neoliberal de la economía profundizó la segmentación o la dualidad de los mercados de trabajo entre países y al interior de cada país. Por otro lado, permitió que tanto el pool de mano de obra calificada como el pool de mano de obra no calificada pudiesen ser reclutados globalmente, la primera, predominantemente a través de la fuga de cerebros (brain drain) y de la subcontratación (outsourcing) de servicios técnicamente avanzados; la segunda predominantemente a través de la deslocalización de las empresas y también a través de la inmigración muchas veces clandestina. La disponibilidad global de mano de obra calificada hizo que la inversión de los países centrales en la universidad pública bajara de prioridad y se volviera más selectiva en función de las necesidades del mercado (De Sousa Santos, 2004: 18).

Además, las becas han sido sustituidas por los préstamos estudiantiles, por los continuos "problemas" que conlleva sostener un sistema de becas dentro de un recorte presupuestario ${ }^{7}$.

Todas estas características hacen preguntarse las diferencias entre una educación privada y una pública; ya que las universidades públicas comienzan asemejarse en funcionamiento a las universidades privadas, cuya inclusión refiere más al consumo ${ }^{8}$, su currícula está pensada en las necesidades de mano de obra del mercado y su presupuesto es recortado, por lo que existe un desfinanciamiento de la investigación ${ }^{9}$, la acción social y de los programas o

$7 \quad$ En varias ocasiones más que recortes es un reacomodo presupuestario hacia las partidas más rentables. Lo que provoca que aquellas personas que quieran realizar trabajo de campo se refugien en trabajos teóricos o históricos que requieren de menos presupuesto pero implican un menor acercamiento y trabajo conjunto con comunidades.

8 Necesidad de estudiar o adquirir un bien por el cual se paga, aunque sea mediante una deuda.

$9 \quad 0$ se financian solo ciertos temas, que son los que se abren a concurso por oNGs, agencias 
proyectos que son menos "rentables", aunque son los que presentan un mayor compromiso social con la realidad de las comunidades.

Pero romper con este modelo de desarrollo mercantil no es posible mientras domine las relaciones de eficiencia, calidad y responsabilidad definidas en términos de mercado, de esta forma el modelo educativo político y pedagógico no tiene cabida.

Las universidades públicas entran dentro de un modelo empresarial educativo cuando lo más importante es maximizar la rentabilidad. Es por eso que dentro de este modelo cabe rescatar la reflexión sobre la importancia de la acción social, ya que se convierte en un espacio diferenciador de la educación privada $y$ dentro de los parámetros del mercado de la educación pública, cuyo principio y objetivo primordial implica un compromiso con la realidad de las comunidades $y$ de esta manera contribuir con la transformación social.

\section{LA ACCIÓN SOCIAL EN UNIVERSIDADES PÚBLICAS}

¡Declárate dueño de tu propio cuerpo, estómago, casa, barrio, fábrica y escuela y actúa después! Mark Lammerink

La acción social es definida por la Vicerrectoría de Acción Social (vas) de la ucR como "la actividad sustantiva que integra y realimenta permanentemente a la Universidad con la comunidad nacional e internacional, para poner a su servicio la capacidad académica institucional, con el propósito de lograr, en conjunto, las transformaciones requeridas para el mejoramiento de la calidad de vida" (s.f.). Esta definición otorga especial atención a las transformaciones para mejorar las condiciones de vida, pero la pregunta que surge es si busca mejorar la calidad de vida de las personas ¿Se

extranjeras, instituciones internacionales, entre otras. Son estas las que definen los términos, las formas, los temas, lo cual desemboca en una pérdida de la autonomía en la discusión, sobre todo de las conclusiones, ya que por lo general en los términos controla de antemano las mismas (De Sousa Santos, 2011). busca que exista una vida digna, equitativa y justa para todos $y$ todas?

Se observa entonces a la acción social como una herramienta epistemológica, política y metodológica que busca modificar la realidad a través de la participación en un proceso organizado y que además construye conocimiento (Chávez y Daza, 2003), gracias a la labor conjunta de una comunidad o grupo organizado $y$ la labor de investigadores o investigadoras.

De esta manera, tal y como lo plantea Park, la acción social "surge como una estrategia para dar poder a los sectores que han sido excluidos institucionalmente de participar en la creación de una nueva sociedad, con el fin de que aquellos puedan asumir las tareas necesarias para lograr mejores condiciones de vida" (1992: 136), implica tanto analizar la realidad en que se inscribe el proceso político, así como, definir acciones concretas a realizar para resistir y solucionar los problemas que enfrentan las comunidades.

La acción social entonces refiere a un diálogo entre la reflexión y la acción (Nayibe y León, 2005), que permite la construcción a partir de las diversas experiencias de las personas, de distintas formas de aprendizajes o soluciones a los problemas que enfrentan de forma práctica. Es así que la propuesta de acción social parte de una serie de enunciados ${ }^{10}$, entre los que se destacan a continuación.

\section{A) TOMA POSICIÓN FRENTE A LA DESIGUALDAD Y LA EXCLUSIÓN}

Las sociedades actuales enfrentan el reto de resistir a un sistema extractivo y acaparador de los recursos (Giarraca y Teubal, 2011 y De Sousa Santos, 2004 y 2006) que convierte el tiempo de las personas en jornadas laborales $y$ el espacio de las comunidades en "industrias para el desarrollo", prohibiendo el uso de los recursos necesarios para la subsistencia humana a menos que se conviertan en mercancía que

10 Los siguientes enunciados son parte del cuerpo textual sobre la acción-social, pero también son acordes a la experiencia durante la ejecución del proyecto con las mujeres de AMUDA; es por este motivo que se destacan estos enunciados. 
se pueda adquirir como una transacción dentro del mercado.

Es así, como existen procesos de pauperización de las condiciones de vida, a los que un gran número de comunidades son sometidas (Ortecho, 2011) como consecuencia de un sistema desigual y de exclusión, expresadas en un conjunto de normas, valores o tratados, creados con el fin de promover el "desarrollo". En las comunidades, estos tienen expresiones locales de control político, así como por ejemplo, una piñera puede apropiarse de grandes extensiones de tierra, destruir grandes extensiones de bosque y utilizar grandes cantidades de agroquímicos, amparada por las reglas del libre mercado.

Las comunidades enfrentan problemas de tenencia de tierra, de contaminación del agua $y$ del aire, amenazas de desalojo, condiciones laborales de explotación, pocas fuentes de trabajo, pérdida de la seguridad alimentaria y de los espacios comunales, esto sumado al poco tiempo disponible para realizar sus actividades cotidianas fuera del trabajo, provoca que los procesos organizativos resulten muy complicados de llevar a cabo.

La resistencia en contra de la desigualdad y la exclusión social parte de la organización y es ahí donde la acción social se convierte en una perspectiva de trabajo útil, para la búsqueda de soluciones a estos problemas y así brindar un acceso verdadero a los recursos que han sido arrebatados por el sistema económicopolítico. En un proyecto de acción social se toma posición ante esta realidad rompiendo con "técnicas neutrales que dejan a las comunidades como víctimas de la explotación científica" (Fals Borda, 2009: 259).

\section{B) NO ES UN PROCESO DE ACAPARACIÓN DEL CONOCIMIENTO, ES UN PROCESO PARTICIPATIVO}

Al tomar posición frente a esta realidad, las y los investigadores también son actores dentro del entramado social, como tales conviven con las comunidades el sentimiento de despojo y no deberían reproducir las prácticas a las que se están enfrentando.
Si "dentro del contexto de las economías políticas modernas, donde la apropiación de riqueza y privilegios por parte de quienes sustenten el poder se realiza a expensas de los que no los tienen" (Park, 1992: 139), los y las investigadoras no deben realizar lo mismo con el conocimiento, este debe ser compartido por todos y todas, no solo con el fin de acaparar el conocimiento de las comunidades para realizar una "investigación académica", sino para proponer caminos a seguir:

El verdadero investigador en este caso no es el investigador tradicional quien, como un experto técnico, se relaciona con los "sus jetos" de la investigación (respondedores a un cuestionario, entrevistados, participantes en un experimento) solo como objetos de investigación, o como fuente de información. Más bien son las personas comunes con problemas por resolver las que colaboran con el investigador con el fin de conocer las dimensiones de la opresión, las contradicciones estructurales $y$ las potencialidades transformadoras de la acción colectiva. Esta es la parte participativa" (ídem: 140).

Esto hace que la acción social sea la búsqueda colectiva de soluciones a los problemas locales, que tienen su origen como parte de un sistema macro, mediante una reflexión conjunta que lleve a propuestas de resistencia.

Esta dimensión participativa requiere de organizarse, comunicarse, trabajar en conjunto y de respetar las experiencias de todos $y$ todas, de manera que estas estrategias de resistencia se dirijan a la concientización y a la acción (Chávez y Daza, 2003). Esto siempre respetando las particularidades de cada proceso, donde el objetivo es crear un espacio horizontal y participativo.

\section{C) LA ACCIÓN SOCIAL RESPETA LOS DISTINTOS CONOCIMIENTOS}

$\mathrm{Al}$ ser un proceso eminentemente participativo, se debe respetar las distintas formas de conocimiento, rompiendo con la idea de que 
el "técnico" es el único que sabe, así realmente las comunidades serán parte del proceso y no solo actores pasivos a la espera de las decisiones de aquellas personas que tienen el "conocimiento": "la gente posee conocimientos valiosos para solucionar problemas. Estos conocimientos están adaptados a las circunstancias locales, al contexto $y$ a la cultura local" (Lammerink, 1999: 43).

Es así como, los distintos conocimientos, no solo el científico, se vuelven necesarios (De Sousa Santos, 2004 y 2006), el saber local, la sabiduría de los pueblos, el conocimiento de la tierra de las y los agricultores, entre otros. Todos estos conocimientos son válidos y ninguno es más importante que otro.

Se parte del postulado de "ecología de saberes", expuesto por De Sousa Santos cuando se refiere a que "no solo hay muy diversas formas de conocimiento de la materia, la sociedad, la vida y el espíritu, sino también hay muchos $y$ muy diversos conceptos de lo que cuenta como conocimiento $y$ de los criterios que pueden ser usados para validarlo" (2010: 50); por lo que esta ecología, sería una contraespitemología que se inserta dentro de la diferencia entre creencias e ideas; intentando romper con que estas últimas son exclusivas del mundo de la filosofía y la ciencia.

Se trata de reconocer todos los saberes, de manera que se utilicen como punto comparativo desde la experiencia transformadora, de manera que respeta la diversidad de conocimientos, cosmovisiones, saberes, creencias y no solo parte del conocimiento universitario positivista, como en algún momento promulgaban las mismas universidades públicas, rompiendo con la idea del experto investigador, ya que todas y todos somos las $y$ los investigadores $y$ en conjunto a partir de la diversidad de saberes se buscan las respuestas o los caminos a seguir.

La acción social propone un intercambio entre quienes poseen el conocimiento científico y la población que posee "otros" tipos de conocimiento, como saberes sociales, saberes populares o saberes artísticos. Además, esta propuesta se convierte en un intento de las universidades por mantener un modelo cercano a las comunidades y "comprometido" con estas, en el contexto de crisis relacionada con una coyuntura socioeconómica de mercado.

La idea de que todos los saberes tienen la misma importancia, es el principio de escucharnos todos y todas, ya que no existe ningún intercambio entre saberes sino se realiza mediante una actitud de escucha de este otro saber.

\section{D) NO ES LIDERAR EL PROCESO}

De la mano con la idea de que todos los saberes deben comunicarse entre sí, se encuentra con un principio fundamental - tanto de la acción social como del modelo de universidad pública- el cual consiste en que ningún conocimiento está por encima de otro; por lo tanto, ningún saber, ningún grupo de personas lidera a otros, sino se construye en conjunto.

Este es un problema serio, debido a que grupos organizados llegan a "aceptar el juicio de quienes tienen un estatus "superior", aunque este juicio este dirigido contra ellos" (Lewin, 1992: 21), reproduciendo el modelo educativo neoliberal, donde quien es dueño del conocimiento válido es quien lidera.

La propuesta de la acción social, pero también en general, del modelo de universidad pública, debe romper con estos esquemas, ya que se trata de un proceso participativo donde se incorpore a movimientos sociales o grupos organizados que establezcan su propias normas bajo los ideales de una sociedad más "justa", a partir de los conocimientos de los mismos grupos y de una sistematización colectiva del conocimiento existente, convirtiendo a los y las involucradas en sujetos "activos" del proceso (Lammerink, 1999).

Las personas involucradas aportan "su conocimiento del problema y llegan colectivamente a una nueva solución. El proceso es participativo y el conocimiento resultante es técnico en naturaleza" (Park, 1992: 142).

\section{E) LA ACCIÓN SOCIAL BUSCA LA TRANSFORMACIÓN SOCIAL}

La perspectiva de trabajo que plantea la acción social y el modelo de universidad pública al que se apuesta, deberían buscar la transformación de las condiciones existentes 
de exclusión y explotación. Al menos este debe ser el objetivo que se busca al realizar investigaciones desde la universidad en conjunto con las comunidades. Este es uno de los ideales de la creación de universidades públicas, a pesar de como anteriormente se observó, es uno de los rubros que se ha desatendido por el avance de las políticas emprendedoras del Estado, que buscan financiar solo lo que es "útil" para el mercado.

En esencia, estas técnicas vienen a constituir un método especial, el método de estudio-acción, cuyo objeto es aumentar la eficacia de la transformación política y brindar fundamentos para enriquecer las ciencias sociales que coadyuven al proceso (Fals Borda, 1973: 52).

El modelo educativo y los fines de este, deberían buscar la transformación social y por lo tanto, la forma de crear conocimiento, desde "los oprimidos" (Alvarado, 2007), finalmente, es a ellos y a ellas, quienes se les niega el acceso a la educación superior con estas políticas, son a ellos y a ellas quienes se les dice que su conocimiento no es válido y al mismo tiempo, son quienes viven día a día los residuos de un sistema excluyente y despojador, llevándolos a organizarse como camino para lograr esta transformación social. Lo anterior implica evaluar el quehacer de la educación superior y por lo tanto, el papel de las universidades públicas, hasta los problemas locales que atañen directamente la vida cotidiana de las personas, como la tenencia de tierra, la contaminación del agua y del aire, la explotación laboral, la violencia física y psicológica del sistema, entre otras.

El recuperar la identidad de los pueblos, su autonomía y autodeterminación que ha sido "aplastada" por una cultura globalizante (Falla, 1998) de prácticas deshumanizantes como la explotación y la exclusión, solo se consigue si la investigación, sea desde el ámbito que sea, está pensada para aportar a una sociedad más justa donde el acceso a los recursos sea equitativo, incluso la educación.

La experiencia de trabajo con un grupo de mujeres de Guácimo, hizo reflexionar sobre la importancia de la acción social, tal y como se ha definido anteriormente, para aportar en la resistencia de los efectos del neoliberalismo en las comunidades costarricenses. De ahí la necesidad de que existan las condiciones y el compromiso institucional de las universidades públicas para desarrollar este tipo de proyectos ¿Es realmente un objetivo de las universidades públicas de hoy el defender este compromiso? ¿Cómo afrontar las políticas universitarias enfocadas en lo "útil" para el mercado desde las propuestas que no generan una venta de servicios, pero sí un vínculo Universidad-Sociedad?

Las respuestas a estas y muchas más preguntas, solo se podrán construir a través de un trabajo en conjunto de aquellas personas que crean en un modelo educativo universitario comprometido socialmente, donde las currículas puestas al servicio del mercado no son lo principal y más bien contradicen la misma finalidad de una universidad pública.

A continuación, se hará referencia a la experiencia concreta del trabajo con AMUDA, grupo de mujeres con las que se trabajó por casi 2 años.

\section{"CUANDO UNA LUCHA POR SUS SUEÑOS, DE VERDAD LOS ALCANZA"}

En noviembre de 2010, como complemento de un proyecto que se desarrolló en el Instituto de Investigaciones Sociales (IIS) de la Universidad de Costa Rica (ucR) sobre la temática de Bienes Comunes, se realizó un encuentro con dirigentes comunales de Costa Rica involucrados e involucradas en conflictos socioambientales. Una de las conclusiones más contundentes de esta actividad fue el reconocimiento de la importancia de la organización comunal como mecanismo de defensa o de resistencia a los impactos del modelo neoliberal, específicamente, en el tema de los recursos naturales; sin embargo, también se reconoció las dificultades que enfrentan para organizarse.

Con la intención de aportar en esta línea, es que se propuso el proyecto de acción social "Apoyo al fortalecimiento de la organización comunal amuda para la defensa de los recursos naturales". amuda significa Asociación de Mujeres por el Desarrollo de África y se localiza en 
Guácimo, Limón de Costa Rica, la cual se encontraba en una crisis fuerte, precisamente por enfrentar el conflicto de organización.

La idea del proyecto fue apoyar el proceso de organización a partir de la construcción de una memoria histórica de AMUDA, con la cual se planificó en conjunto, un calendario de actividades para realizar acciones que apoyen a la consolidación de la asociación, así como, talleres informativos en distintas áreas.

Los objetivos del proyecto fueron: construir una memoria colectiva de la organización comunal, facilitar los espacios de reflexión que sean necesarios para la organización comunal, elaborar talleres de formación y capacitación con las integrantes de amuda $y$ finalmente, elaborar una reflexión que se comparta con ellas a manera de cierre. Paralelo a este trabajo se avanzó en trabajos puntuales en la finca AMUDA.
De manera general, Guácimo es una cantón de la provincia de Limón donde habitan 41 266 personas; 20648 mujeres y 20618 hombres (Instituto Nacional de Estadísticas y CensosINEc, 2011), con una extensión de 576,5 $\mathrm{Km}^{2}$. De acuerdo al Índice de Desarrollo Humano Cantonal, en el 2005 se encontraba en el puesto 68; en el 2009 en el puesto 69 y para el 2012 en el puesto 71 (Programa de las Naciones Unidas para el Desarrollo-pnud, 2012).

La economía está ligada al sector agroindustrial (tabla 1) por el monocultivo de la piña, el cual ha venido en aumento desde finales del siglo pasado. Según datos del último censo, en Guácimo, 14633 personas mayores 15 años están ocupadas o empleadas, lo que representa cerca del 35\% de la población total; sin embargo, personas mayores de 15 años hay 29 059, por lo que el número de personas desempleadas o subempleadas es de $14426^{11}$ (InEC, 2012).

TABLA 1

PORCENTAJE DE POBLACIÓN OCUPADA

POR SECTOR ECONÓMICO Y DISTRITO

CANTÓN DE GUÁCIMO, LIMÓN

2012

\begin{tabular}{|c|c|c|c|c|}
\hline \multirow{2}{*}{ DISTRITO } & \multirow{2}{*}{$\begin{array}{l}\text { POBLACIÓN DE } \\
15 \text { AÑOS O MÁS } \\
\text { OCUPADA }\end{array}$} & \multicolumn{3}{|c|}{ PORCENTAJE DE POBLACIÓN OCUPADA } \\
\hline & & SECTOR PRIMARIO & $\begin{array}{c}\text { SECTOR } \\
\text { SECUNDARIO }\end{array}$ & $\begin{array}{c}\text { SECTOR } \\
\text { TERCIARIO }\end{array}$ \\
\hline Guácimo & 12919 & 31,8 & 16,1 & 52,1 \\
\hline Mercedes & 1337 & 29,2 & 11,0 & 59,8 \\
\hline Pocora & 4631 & 42,0 & 12,7 & 45,2 \\
\hline Río Jiménez & 6077 & 71,5 & 5,5 & 23,0 \\
\hline Duacarí & 4095 & 79,5 & 5,7 & 14,8 \\
\hline TOTAL & 29059 & 48,1 & 11,7 & 40,1 \\
\hline
\end{tabular}

Fuente: INEc. Censo 2011.

11 En esta cifra se contabilizan las personas mayores de 15 años que se encuentran pensionadas, estudiantes y que se dedican a las tareas del hogar, junto con las personas que se encuentran desempleadas. 
Es importante destacar el crecimiento urbanístico que se ha consolidado en los distritos centrales de Guácimo y Mercedes, muchos barrios se han construido por medio del Instituto Nacional de Vivienda y Urbanismo (INvU) para relocalizar personas de barrios urbanomarginales del Valle Central. Esta práctica ha fomentado mano de obra barata para la expansión piñera; asimismo, esto ha permitido que las empresas puedan estar despidiendo varios empleados en plazos cortos y contratar nuevos, evitando el pago de garantías laborales como el aguinaldo y el seguro social. El crecimiento urbanístico en la zona también ha provocado un aumento en el sector terciario, ligado al aumento de comercios en la zona, en la cual se suma algunos proyectos de ecoturismo y de turismo.

La primacía del sector primario en el cantón se debe a los monocultivos que han caracterizado la historia de la región; incluso, los pueblos como Guácimo y aledaños fueron construidos junto con el ferrocarril en 1884, para así sacar los productos de exportación (primordialmente, banano y café). El responsable de la construcción del ferrocarril en el Atlántico costarricense fue el norteamericano Minor Keith Cooper, quien a cambio recibió grandes extensiones de tierra en esta región; gracias a las cuales fundó la empresa Tropical Trading and Transport Company luego renombrada a United Fruit Company ${ }^{12}$.

Dichas tierras fueron cultivadas principalmente de banano $y$ alrededor de los cultivos se fueron creando asentamientos de trabajadores quienes llegaban a trabajar en el mono-

12 A partir de lo logrado en Costa Rica, se inicia el proceso de expansión hacia El Salvador y Honduras; y luego de la fusión con la empresa competidora y el cambio de nombre, la empresa tuvo tierras en prácticamente toda América Central (excepto Panamá), Colombia, Ecuador, Chile, Perú, Cuba y otras islas en el Caribe. Se le vincula con masacres (masacre del banano, en Colombia), golpes de Estado (a Jacobo Árbenz en Guatemala por intentar expropiar grandes extensiones de tierra), pago de sobornos $y / 0$ a paramilitares (casos en Honduras y Colombia) y se les vincula a la contaminación ambiental en sus plantaciones, violaciones a derechos laborales de sus trabajadores y a permitir la entrada de cocaína a distintos países (Gallagher y McWhirter, 1998). cultivo, a pesar de la salida de la empresa ya a mediados del siglo xx; esto produjo la creación de una serie de asentamientos campesinos que recuperaron una porción de la tierra y la reconversión de la agricultura en la región; es así como para la década de los 70 comienzan a mostrarse el interés en el cultivo de la piña, ya para esa década Pineapple Development Corporation (PINDECO), subsidiaria de la empresa transnacional Del Monte, poseía el 65\% de la producción de piña (Acosta, 2008), desde esa década la producción piñera ha crecido desmedidamente acelerando la pérdida de cultivos tradicionales (ñame, yuca, maíz, entre otros), una serie de problemas ambientales y conflictos sociales.

En este panorama, las comunidades de Limón se caracterizan por una exclusión social debido a que:

... desde su constitución con la creación de un enclave neocolonialista de producción agrícola que modeló el sistema productivo y las relaciones sociales, ligándola de forma desventajosa a los dictados del mercado mundial. Más tarde, porque aún después de la salida de la United Fruit Company del área, Limón siguió siendo destinado a la producción agrícola para la exportación y a los servicios de transporte, almacenaje y flete desde el puerto, sirviendo así a las necesidades de las empresas nacionales y transnacionales $y$ del gobierno central, pero no a las de sus habitantes (Caamaño, 2006: 164)

De esta forma, estas comunidades al día de hoy presentan una serie de problemáticas con las que conviven sus pobladores; problemas de salud relacionados con el alto uso de agroquímicos en las plantaciones y sobre los mantos acuíferos, problemas ambientales por la pérdida de bosques, así como de especies de flora y fauna de la zona, problemas de tenencia de tierra que atenta contra la producción tradicional campesina que brinda seguridad alimentaria y estabilidad económica, problemas sociales como la pobreza por injustas condiciones laborales $y$ pocas fuentes de trabajo distintas al monocultivo $y$ un hacinamiento por 
la expansión de procesos de urbanización por la llegada de mano de obra barata a la región para abastecer las necesidades de las empresas exportadoras. En este panorama, más un olvido de las instituciones estatales en la región para el mejoramiento de las condiciones de vida, hace que hoy en Limón, el desarrollo capitalista $y$ colonialista tenga un peso importante en la cotidianidad de la provincia y de sus cantones.

Es así como, en este contexto se enmarca este proyecto, ya que una forma de enfrentarse a este modelo de desarrollo es la organización comunal, como una forma de resistir en el contexto de políticas coloniales y capitalistas que no benefician a las comunidades.

AMUDA nace en 1997 por iniciativa de un grupo de mujeres de la comunidad de África, Guácimo, que buscaban un espacio y la oportunidad de aportar esfuerzos a la preservación del medio ambiente, en particular, el bosque tropical húmedo, desarrollando tecnologías alternativas y amigables con la naturaleza, en beneficio para la comunidad. Entre los valores que sostiene a esta asociación son compartir, dar esperanza a las futuras generaciones, conservar el bosque, el respeto a la naturaleza, solidaridad, seguridad alimentaria, perseverancia, esfuerzo, respeto a las diferentes capacidades y principalmente, la oportunidad de soñar.

AMUDA se conforma como un grupo de mujeres que tiene el interés de organizarse y buscar una forma distinta de convivir con la naturaleza y con la tierra; es por ello que en sus primeros años adquieren un terreno de 3 hectáreas, el cual deciden dejar 2 hectáreas de bosque como "el último pulmón de Guácimo" y en la otra hectárea plantear un proyecto educativo, que logre crear una reflexión sobre la relación naturaleza-comunidad y una crítica al modelo agroindustrial de explotar el suelo $y$ a los $y$ las trabajadoras. De ahí, la importancia para ellas de preservar este bosque tropical húmedo a través de una finca autosostenible y agroecológica, con tecnologías alternativas y brindando oportunidades de educación ambiental, así como, el rescate de sus raíces culturales.

Este proyecto de la finca AMUDA, cobra una especial importancia por ubicarse en una zona en la que en los últimos años enfrenta una problemática ambiental muy severa. La expansión de monocultivos, en particular, la expansión y contaminación piñera, la tala de árboles y la tenencia de la tierra, son algunos de los problemas que caracterizan los conflictos socioambientales que enfrentan la población caribeña.

Durante el trascurso de los años, muchas mujeres abandonaron el trabajo con AмUDA, por distintos motivos como proyectos personales o por falta de tiempo al tener horarios de trabajo que les dificultaba reunirse.

El punto de partida y de llegada, fue trabajar a partir de las necesidades e intereses de las mujeres que participan en Amuda $y$ para ello, se utilizó el enfoque metodológico de la investigación-acción, de acuerdo con la perspectiva de los Bienes Comunes, que no es más que un grito por un nuevo espíritu de comunidad, de anteponer al mercado, el interés del bien común.

Este apoyo se concretó facilitando la reflexión permanente sobre la comprensión de cuáles son las dificultades que enfrentan y cómo hacerles frente, el contexto micro y macro en el que se dan estas dificultades, así como, por medio de visitas a fincas agroecológicas y talleres participativos de información y capacitación con especialistas en los temas específicos que ellas manifestaron de su interés o necesidad. Se parte de complementar el saber de la organización comunal y el saber académico, tal y como lo plantea De Souza Santos (2004), que promueva una nueva convivencia activa de saberes.

En este tiempo se incorporaron nuevas mujeres al grupo, este se amplió y se organizó frente a las estrategias para terminar de pagar la finca; generar algún ingreso, participar en talleres o cursos que alguna institución impartía. Asimismo, se discutió sobre la importancia de organizarse frente al contexto neoliberal en que se vive. Se abrieron espacios para que ellas preguntaran, opinaran $y$ decidieran que hacer con y en la finca. Es así como nace la idea de realizar un proyecto educativo-ambiental, de esta forma se comienza con la búsqueda de materiales y la realización de talleres para conocer 
las implicaciones, las dificultades y las experiencias en este tipo de proyectos.

Durante este tiempo algunas de ellas se separaron del grupo debido a dinámicas internas del mismo o por problemas laborales o familiares, existieron momentos de mucha dificultad, que son parte de los retos que siempre van a enfrentar proyectos de acción social con grupos comunitarios y principalmente cuando se trata de trabajar en el apoyo al fortalecimiento de la organización.

Los principales momentos de tensión durante el proceso, fueron el enfrentar las necesidades económicas por algunas participantes del grupo y problemas de salud de alguna persona de sus familias, así como, la angustia de no contar con el dinero para enfrente a la deuda del pago de la finca. Estas situaciones se contrapusieron a la dinámica del grupo, al tener que establecer como prioridad la atención a estas necesidades, dificultando la participación activa en Amuda. Sin embargo, se contó con la acción, comprensión y solidaridad del grupo para lograr enfrentar estos obstáculos y así poder continuar con el proyecto.

Durante el año $y$ nueve meses que se trabajó con amuda, la ética se sostuvo en el respeto mutuo, precisamente por medio del diálogo de saberes, entre la academia y las experiencias, vivencias y otros saberes. Se planteó un proceso en ambas direcciones, por un lado que permita a las organizaciones romper dependencias $y$ que construyan "comunidad" bajo sus propios conocimientos $y$ principios, acompañados $y$ fortalecidos por conocimientos y espacios que la Universidad pueda construir. Al mismo tiempo que permita a la academia, generar espacios internos de reflexión, sobre dinámicas y realidades sociales que no se logran comprender desde los espacios propiamente académicos, ni con la revisión de textos, sino, compartiendo, escuchando y comprendiendo estas experiencias, vivencias y saberes que se construyen en este tipo de organizaciones.

Es importante destacar que el enfoque metodológico de la investigación-acción permitió cumplir los objetivos en el apoyo al fortalecimiento de AмUDA, porque como plantean
Chávez y Daza, esta metodología facilita la comunicación, el trabajar en conjunto:

... principios que se fundamentan en aceptar la sabiduría popular y el sentido común de cada sujeto, su desarrollo como ser social capaz de comunicar puntos de vista, evocar y compartir experiencias, analizar sus condiciones de vida $y$ concientizar sobre las posibilidades de transformación. La investigación acción se hace con una óptica desde dentro y desde abajo; participar no es una casualidad que se da, sino el derecho de todos a ser sujetos de historia, o sea, protagonistas dinámicos de las actividades que cada grupo traza y lleva adelante (2003: 122).

Cárdenas señala que este tipo de investigaciones participativas tienen el doble propósito de la organización comunal para la toma de decisiones y la "construcción de sujetos políticos con capacidad de organización y movilización social, el fortalecimiento de esquemas internos de organización comunitaria y de toma de decisiones..." (2009: 95). Si bien es cierto, amuda tiene mucho camino por andar para lograr plasmar su proyecto en su finca agroecológica $y$ autosostenible, se puede afirmar que el trabajo realizado durante casi dos años, aportó en esta doble dirección que plantea Cárdenas. amuda es una organización más autónoma y fortalecida que la que se encontró al inicio.

Asimismo, se comparte el planteamiento de Park cuando señala que "la Investigación Acción Participativa (IAP) es un medio para que la gente recupere su capacidad de pensar por sí misma y de innovar, así como también para recordar su historia y revivir su cultura para la recreación de una vida auténtica... La IAP es tanto un proceso de recuperación como de descubrimiento" (1992: 156). De esta manera se sintetiza uno de los resultados más fuertes del trabajo con AMUDA, ilustrado con las palabras de las participantes del proyecto:

- Por eso el pesimismo, las circunstancias, la falta de recursos, el no tener comunicación y el miedo quedaron superados y al fin su ser, está preparado para 
salir a surcar los cielos. Y nunca olvidar esas voces tanto masculinas como femeninas, que le han brindado amor, esperanza y fortaleza dando una base sólida para poder dar el paso de romper ese capullo y ver su futuro con alegría y sin miedo. Pero siempre preparada para enfrentar las adversidades que siempre existirán pero ya confiada en que sí se pueden superar. amuda sabe que... "Cuando uno lucha por sus sueños de verdad los alcanza".

El aporte brindado en su empoderamiento como organización pero también como personas, así como, el haber contribuido a la oportunidad de soñar, de soñarse como organización fuerte, capaz de enfrentar cualquier obstáculo y lograr plasmar su proyecto. AmuDa representa la esperanza en la organización comunal como mecanismo de resistencia a los impactos del neoliberalismo y poder construir una sociedad menos desigual y más justa para todas y todos. Resaltando las palabras de Eduardo Galeano, la utopía sirve porque nos permite caminar.

\section{CONCLUSIONES}

Ante la crisis institucional, como la llama De Sousa Santos (2006), donde nuestras universidades públicas creadas bajo el principio de autonomía, actualmente son administradas $y$ orientadas como si fueran empresas, con valores característicos del mundo empresarial, como por ejemplo, eficacia, productividad, la creación de mercados de servicios universitarios o venta de servicios, como también se conoce. Es urgente el fortalecimiento de la acción social como compromiso ético político de nuestras universidades con nuestras comunidades, con la sociedad: "una universidad de proximidad, de un bien público que realmente sea accesible, con calidad, y que esté relacionado con los problemas de la sociedad donde esté ubicada" (De Sousa Santos, 2006: 59).

Desde esta experiencia, la acción social $y$ específicamente, la investigación-acción es un excelente planteamiento para plasmar este compromiso ético-político, porque facilita, por un lado, empoderar a las personas que encuentran en la organización comunal la forma para enfrentar y resistir los impactos del neoliberalismo, entre los cuales se pueden mencionar, la extracción de la naturaleza, el individualismo, la desigualdad y las injusticias. Pero, también por otro lado, la investigación-acción, con su diálogo de saberes, aporta a la suma de conocimientos y experiencias que contribuya a la construcción de una sociedad más solidaria y humana. En otras palabras, la investigaciónacción es un encuentro con la esperanza, pero tal y como la conceptualizan José Martí y Paulo Freire, no como acto de fe, sino como compromiso en y con la sociedad para la transformación de esta.

La investigación-acción es una práctica que permite no solo generar conocimiento desde la fuente primaria, sino crear vínculos con los distintos actores sociales y la devolución de los resultados.

Esto obliga a replantearse cuál es el modelo de universidad pública que se quiere y cuál existe, inevitablemente se cuestiona si son el mismo o si se han distanciando; mientras se aboga por una universidad comprometida con la transformación social y con las comunidades, se pregunta si la Universidad está más comprometida con asegurar las necesidades de mano de obra del mercado.

Esta reflexión busca aportar en este debate, planteando la importancia de que la Universidad sea parte de esta transformación, colaborando con la organización comunitaria (no liderándola), aportando al debate crítico de la organización social a través de una visión sobre los problemas sociales y del sistema excluyente que los origina.

$\mathrm{Al}$ igual que las comunidades enfrentan problemas por una serie de políticas de corte neoliberal que atenta contra la dignidad de la vida, en las universidades públicas existe un desfinanciamiento hacia los intentos que como la acción social, se proponen aportar en la transformación hacia la búsqueda de una equidad basada en el bien común. La defensa del modelo de universidad pública y acción social, tal y como se concibió en sus inicios, se convierte en un imperativo en la actualidad. 
En este sentido, la experiencia de trabajo con amuda significó un encuentro con la esperanza, en tanto que a pesar de todas las limitaciones personales y sociales (el contexto en el que se encuentran inmersas) que enfrentan, siempre su actitud positiva y acertiva para enfrentarlas, posibilitó la oportunidad de soñar. Soñar en una sociedad más solidaria y justa, menos desigual y armoniosa con la naturaleza.

\section{BIBLIOGRAFÍA}

\section{LIBROS}

Chávez, Guadalupe y Daza, Juan Carlos. "Reflexión metodológica sobre la aplicación concreta de la Investigación Acción Participativa (IAP) en contextos rurales del estado de Colima”. Estudios sobre las Culturas Contemporáneas Ix (17). México. Universidad de Colima, 2003: 115-146.

De Sousa Santos, Boaventura. La Universidad del siglo xxi. Para una reforma democrática y emancipadora de la Universidad. México: Centro de Investigaciones Interdisciplinarias en Ciencias y Humanidades Universidad Nacional Autónoma de México ( UNAM), 2004.

De Sousa Santos, Boaventura. Renovar la teoría crítica y reinventar la emancipación social. Buenos aires, Argentina: Consejo Latinoamericano de Ciencias Sociales (clacso), 2006.

De Sousa Santos, Boaventura. Descolonizar el saber, reinventar el poder. Montevideo, Uruguay: Ediciones Trilce, 2010.

De Sousa Santos, Boaventura. "Desafíos a la sociología en la universidad del siglo xxı". Cuadernos de Sociología 11. Escuela de Sociología, Universidad de Costa Rica, 2011: 27-67.

Fals Borda, Orlando. Una sociología sentipensante para América Latina. Bogotá, Colombia: Siglo del Hombre Editores-Consejo Latinoamericano de Ciencias Sociales (clacso), 2009.
Lewin, Kurt. "La investigación-acción y los problemas de las minorías". La investigación-acción participativa: inicios y desarrollos. María Cristina Salazar (coord.). Editorial Popular-Organización de Estados Iberoamericanos para la Educación, la Ciencia y la Cultura (oEI)-Sociedad Estatal Quinto Centenario, 1992: 15-26.

\section{PUBLICACIONES PERIÓDICAS}

Alvarado, Miguel. "José Martí y Paulo Freire: aproximaciones para una lectura de la pedagogía crítica". Revista Electrónica de Investigación Educativa 9 (1). 2007. En: < http://redie.uabc.mx/index.php/redie/ article/view/157/271> [Consultado 04 de julio de 2013].

Caamaño, Carmen. "Desarrollo capitalista, colonialismo y resistencia en Limón". Anuario de Estudios Centroamericanos 32. Universidad de Costa Rica, 2006: 163193.

Cárdenas, Gloria. "Investigación participativa con agricultores: una opción de organización social campesina para la consolidación de procesos agroecológicos”. Revista Luna Azul 29. Universidad de Caldas, 2009: 95-102.

Falla, Ricardo. "Investigación y acción social: claves para una alianza". Revista Envío 200. Universidad Centroamericana (UCA), 1998.

Fals Borda, Orlando. "Reflexiones sobre la aplicación del método de estudio-acción en Colombia”. Revista Mexicana de Sociología 35 (1). Universidad Nacional Autónoma de México, 1973: 49-62.

Giarracca, Norma y Teubal, M. "Disputas por los territorios y recursos naturales: el modelo extractivo". Revista ALASRU Nueva Época 5. Asociación Latinoamericana de Sociología Rural, 2011: 113-133.

González, Yamileth. "Costa Rica". Historia de las universidades de América Latina. Rodríguez, Gisela (ed.). Colección udUaL: 1999.

Lammerink, Mark. "Educación popular y la Investigación Acción Participativa. 
Propuestas desafiantes para Europa". Kikiriki. Cooperación educativa 53. 1999: 39-44.

Nayibe, Lilliam y León, Aníbal. "Perspectiva crítica de Paulo Freire y su contribución a la teoría del currículo". EDUCERE. Revista venezolana de Educación 9 (29). Universidad de los Andes, 2005: 154-169.

Ortecho, Mariana. "Cruce de paradigmas y complejización de abordajes en procesos de investigación- acción social". Revista de Ciencias Sociales 133-134 (III-IV). Universidad de Costa Rica, 2011: 41-56.

Park, Peter. “¿Qué es la investigación-acción participativa? Perspectivas teóricas y metodológicas". La investigación-acción participativa: inicios y desarrollos. María Cristina Salazar (coord.). España. Editorial Popular, Organización de Estados Iberoamericanos para la Educación, la Ciencia y la Cultura (oEI) y Sociedad Estatal Quinto Centenario, 1992: 135-174.

\section{TEXTOS ELECTRÓNICOS}

Gallagher, Mike y McWhirter, Cameron. "Chiquita SECRETS Revealed". Cincinnati Enquirer. Mayo 1998. En: <http://www. mindfully.org/Pesticide/chiquita/> [consultado el 3 de julio de 2013].

González Casanova, Pablo. "La autonomía universitaria, hoy". La Jornada. 12 de octubre de 2006. En: <http://www. jornada.unam.mx/2004/10/12/018a1pol. php> [consultado el 4 de julio de 2013].

Instituto Nacional de Estadísticas y Censos -Inec. Censo 2011. Costa Rica: Instituto Nacional de Estadísticas y Censos-inec, 2012. En: <http://www.inec.go.cr/ Web/Home/Generador Pagina.aspx> [consultado el 2 de julio de 2013].
Programa de las Naciones Unidas para el Desarrollo (PNUD). Atlas del Desarrollo Humano Cantonal de Costa Rica 2012. PNUD y Universidad de Costa Rica, 2012. En: <http://www.pnud.or.cr/mapacantonal/> [consultado el 2 de julio de 2013].

Vicerrectoría de Acción Social. ¿Qué es Acción Social? Universidad de Costa Rica, s.f. En: <http:/accionsocial.ucr.ac.cr:8080/ accion-social> [Consultado el 24 de mayo de 2013].

OTROS

Acosta, Esteban. "La producción piñera en Costa Rica: un análisis integral de sus implicaciones en el cantón de Guácimo, Limón”. [Proyecto de graduación para obtener el título de Ingeniero Agrónomo con el grado de Licenciatura en Ciencias Agrícolas de la EARTH]. Limón, Costa Rica: 2008. En: <http://usi.earth.ac.cr/glas/sp/ ColeccionVirtual/pdf/PG67-2008_ acostaE\%5B1\%5D.pdf> [Consultado el 4 de julio de 2013].

Pérez, María y Meoño, Rita. Trabajo comunal universitario: la conquista de un derecho. San José, Costa Rica: Vicerrectoría de Acción Social de la Universidad de Costa Rica, 2007.

Universidad de Costa Rica. Estatuto orgánico de la Universidad de Costa Rica. Consejo Universitario de la Universidad de Costa Rica, 1974.

Fecha de ingreso: $12 / 02 / 2014$

Fecha de aprobación: 08/08/2014 


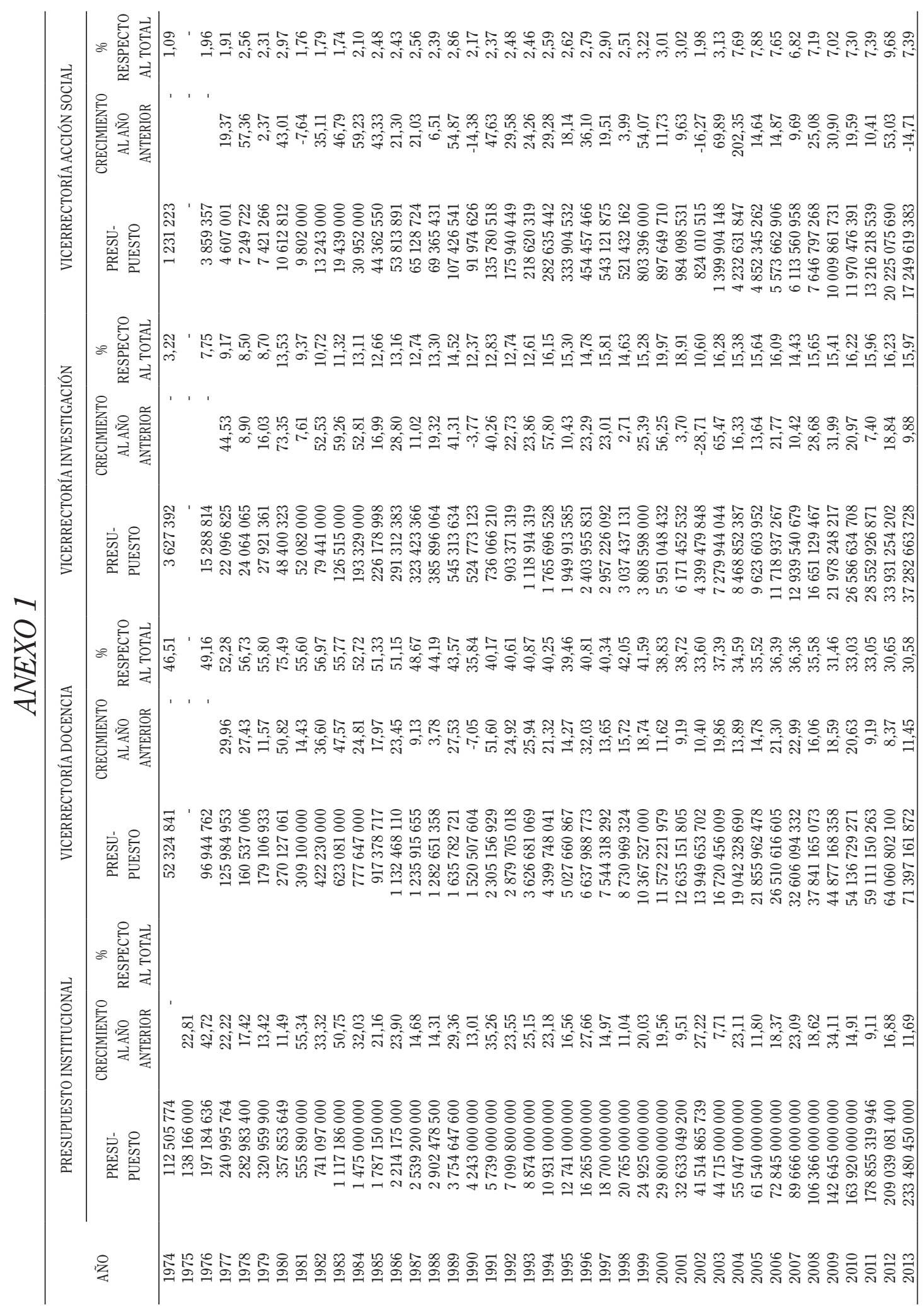

(c) (1) (-) Rev. Ciencias Sociales Universidad de Costa Rica, 148: 49-66 / 2015 (II). (ISSN: 0482-5276) 